1

\title{
Numerical study on the limit of power extraction by a dense cross-stream array of wind turbines
}

\author{
Filiberto Tartari ${ }^{*}$, Takafumi Nishino ${ }^{\dagger}$ \\ Centre for Offshore Renewable Energy Engineering, Cranfield University, Cranfield, \\ Bedfordshire MK43 0AL, United Kingdom
}

\section{Abstract}

A numerical study is presented on the upper limit of power extraction by a dense cross-stream array of wind turbines, using 3D Reynolds-averaged Navier-Stokes simulations of flow over porous discs. The main objectives are: (i) to investigate the effect of 'local blockage' due to neighbouring turbines on the limit of power extraction; and (ii) to clarify how this effect compares with the effect of 'local flow acceleration' obtained by staggering the array in the streamwise direction. Some unconventional array configurations with vertical turbine arrangements, following the so-called 'multi-rotor' concept, are also investigated. Results show that the limit of power extraction by a non-staggered array increases moderately with the number of turbines arrayed (about $5 \%$ increase in the power coefficient compared to the Betz limit when 9 turbines are arrayed side-by-side). This power increase due to the local blockage can be enhanced further, but only slightly for the case of 9 turbines, by arranging turbines vertically as well as horizontally. Staggering the array in the streamwise direction may increase the power of downstream turbines due to the effect of local flow acceleration but reduce the power of upstream turbines as the local blockage effect diminishes, resulting in a total power reduction.

Keywords: Blockage effect; Efficiency; Multi-rotor; Staggered array; Wind farm

\footnotetext{
* Present address: Mott MacDonald, Victory House, Trafalgar Place, Brighton BN1 4FY, United Kingdom

${ }^{\dagger}$ Corresponding author. E-mail: t.nishino@cranfield.ac.uk
} 


\section{Introduction}

Nowadays, wind turbines are often clustered in arrays or wind farms to reduce the land use as well as the cost of installation and maintenance. However, the interaction of turbine wakes in such a wind farm often results in not only increased dynamic loads (as a consequence of higher turbulence level) but also a reduced total power production (Sørensen, 2011). It has been demonstrated that the power losses due to the wake effects in grid-like arranged wind farms can be significant, largely depending on the streamwise spacing between adjacent lateral (or cross-stream) rows of turbines. Consequently, the optimisation of turbine spacing and layout in large wind farms has recently become a key research topic in the wind energy sector (Meyers and Meneveau 2012; Porté-Agel et al., 2013; Ghaisas and Archer, 2016; Stevens, 2016; Nishino, 2016).

In this study we investigate a possible enhancement of power production by a dense crossstream array or 'fence' of wind turbines. Specifically, we investigate how the maximum total power produced by a given number of turbines could be increased by arranging the turbines densely but only in the cross-stream direction (so that no turbines are placed in the wake of other turbines). Although such a fence of wind turbines can be deployed on its own, this can also be a constituent element of a large wind farm, i.e. it is possible to deploy several fences of turbines to form a large wind farm. In the latter case, some fences could be located in the wake of other fences, the effect of which is not investigated in the present study. Nevertheless, the local (or fence-scale) flow physics discussed in this paper is of great importance to the performance of multiple-fence wind farms to be considered in future studies.

For almost a century it has been known that the upper limit of power extraction by a single ideal wind turbine rotor is $16 / 27$ (or 59.3\%) of the kinetic power of natural wind passing through the rotor swept area, known as the Betz limit or the Betz-Joukowsky limit (Okulov and van Kuik, 2012). However, recent theoretical and numerical studies on the efficiency of tidal turbines (Garrett and Cummins, 2007; Nishino and Willden, 2012a, 2012b, 2013; Draper and Nishino, 2014) have shown that this upper limit of power extraction may increase significantly when the passage of flow around a turbine is constrained, often referred to as the power increase due to 'blockage effect'. In particular, 
the recent work by Nishino and Willden (2012b, 2013) has highlighted that the blockage effect may arise not only when the flow around a turbine is constrained by physical boundaries (such as windand water-tunnel walls, ground, seabed and sea-surface) but also due to the existence of neighbouring turbines that effectively constrain the passage of flow around each turbine in the case of a fence of tidal turbines. More recently, Nishino and Draper (2015) have explained theoretically and demonstrated numerically that such a 'local' blockage effect due to neighbouring turbines may arise in the case of a fence of wind turbines as well, although the effect seems to be less significant compared to the case of tidal turbines. A similar blockage effect for wind turbines (called 'in-field' blockage effect) has also been investigated experimentally by McTavish et al. (2015).

The main aim of the present study is to better understand the effect of local blockage on the limit of power extraction by a fence of wind turbines. One interesting question to be answered is whether the maximum power of a turbine fence could be further increased by displacing some of the turbines in the fence in the streamwise direction (rather than arranging all turbines perfectly side-byside). This question has been examined recently by Hunter et al. (2015) for the case of tidal turbines, but has not been examined systematically for the case of wind turbines. To address this, we perform three-dimensional (3D) Reynolds-averaged Navier-Stokes (RANS) simulations of flow around several different arrangements of idealised turbines (represented by porous discs) placed near a plane boundary. In addition to conventional cross-stream arrays of wind turbines with each turbine having the same rotor hub height, we also consider some unconventional arrangements where some rotors are placed above other rotors, following the concept of 'multi-rotors' studied recently by Jamieson and Branney (2012), Chasapogiannis et al. (2014) and Manwell et al. (2014).

\section{Methodology}

\subsection{Computational methods}

The numerical simulations are performed using a commercial CFD solver ANSYS FLUENT 15.0, solving 3D incompressible RANS equations numerically based on a finite volume method. The Reynolds stress terms in the RANS equations are modelled using the standard $k-\varepsilon$ model of Launder 
and Spalding (1974). The convective terms in the RANS equations are discretised using the secondorder upwind scheme, whereas the first-order upwind scheme is used for the transport equations of $k$ and $\varepsilon$. The SIMPLE algorithm (Patankar, 1980) is used to solve the mean velocity and pressure fields iteratively.

The wind turbines are modelled using a porous disc model, similarly to the earlier studies on the local blockage effect for wind turbines (Nishino and Draper, 2015) as well as for tidal turbines (Nishino and Willden, 2013). Specifically, each turbine is represented by a stationary permeable disc, which is implemented using the 'porous jump' internal boundary condition in FLUENT. The effect of each disc on the mean flow is considered as a loss of momentum at the disc in the streamwise $(x)$ direction. The change of $x$-momentum flux (per unit disc area) is locally calculated as

$$
M_{x}=K \cdot \frac{1}{2} \rho U_{d}^{2}
$$

where $\rho$ is the density of air, $U_{d}$ is the local (rather than disc-averaged) streamwise velocity at the disc plane and $K$ is the momentum loss factor. In this study we assume that the value of $K$ is uniform across the surface of all discs. Note that this assumption (i.e. prescribing a uniform disc resistance) results in a non-uniform thrust distribution across each disc (since in general the velocity $U_{d}$ is not uniform across the disc). This may appear to contradict the conventional actuator disc theory, which usually explicitly assumes a uniform thrust distribution across the disc. However, a recent study by Draper et al. (2016) has shown that the same theoretical upper limit of power extraction can be generally reached by assuming a uniform resistance; hence the above porous disc model is fairly compatible with the theoretical actuator disc model. For further clarification of the relationship between the numerical porous disc model and the theoretical actuator disc model, see Nishino and Draper (2015) and Nishino (2016).

As noted by Nishino and Willden (2012a), the main advantages of using a porous disc model in this type of numerical study are its generality (i.e. not requiring any particular geometry of turbine rotors), simplicity and compatibility with the actuator disc theory. Since the main aim of the present study is to understand the effect of local blockage on the 'limit' of power extraction by a fence of ideal turbines, the above porous disc model is sufficient for this study. To investigate the effect of 
local blockage on the performance of real turbines, however, we would need to employ a higherfidelity turbine model, such as actuator-line and actuator-surface models (Sørensen, 2011). Also, when some turbines are located in the wake of other turbines and hence the accuracy in the prediction of wake mixing is important, higher-fidelity simulations of turbulent flows, such as Large-Eddy Simulations (LES), would be more desirable than the RANS simulations performed in this study.

\subsection{Computational domain and array configurations}

Following the earlier study by Nishino and Draper (2015), we employ the same size of computational domain in this study; $25 D$ in height, $50 D$ in width and $100 D$ in streamwise length, where $D=100 \mathrm{~m}$ is the disc diameter. The array of discs is positioned at the centre of the domain near the ground (bottom boundary) with a fixed cross-stream gap of $0.5 D$ between each disc (from edge to edge). The vertical gap from the ground to the lowest disc edge is also maintained at $0.5 \mathrm{D}$. Unless specified, all cases investigated in this study are for an array of 9 discs, which results in a very small 'global' blockage ratio (i.e. the ratio of the total area of discs to the cross-sectional area of the computational domain) of $B_{G} \approx 0.006$. As noted by Nishino and Draper (2015), this essentially means that the computational domain is large enough to conclude that the global blockage effect is negligibly small (and hence the power increase observed is due to 'local' flow mechanisms within the array).

Table 1 summarises the array configurations investigated in this study. Also, Fig. 1 shows the three main array configurations (Array-A, Array-B and Array-C). For Array-A, all discs (up to 9 discs) are arrayed only horizontally near the ground (like a conventional lateral array of wind turbines). For Array-A, we consider two different staggered arrangements, namely 'zigzag' and 'Vform' arrangements, as well as the non-staggered (or side-by-side) arrangement. For Array-B, 5 discs are arrayed horizontally near the ground (to form the first row) and 4 discs are arrayed above these 5 discs (to form the second row). For this Array-B, we consider a staggered arrangement like a 'step' (i.e. only the 4 discs forming the second row are shifted downstream) as well as the non-staggered arrangement. For Array-C, 4 discs form the first row, 3 discs form the second row and 2 discs form the third row. 
Table 1. Summary of array configurations.

\begin{tabular}{cccc}
\hline Configurations & Staggered? & Streamwise gap & Number of discs \\
\hline Array-A & No & 0 & $1,3,5,7,9$ \\
Array-A & Yes (zigzag) & $0.5 D$ to $3 D$ & 9 \\
Array-A & Yes (V-form) & $0.5 D$ to $3 D$ & 9 \\
Array-B & No & 0 & 9 \\
Array-B & Yes (step) & $0.5 D$ to $3 D$ & 9 \\
Array-C & No & 0 & 9 \\
\hline
\end{tabular}

136

137

138

(a)

000000000

(b)

0000

(c)

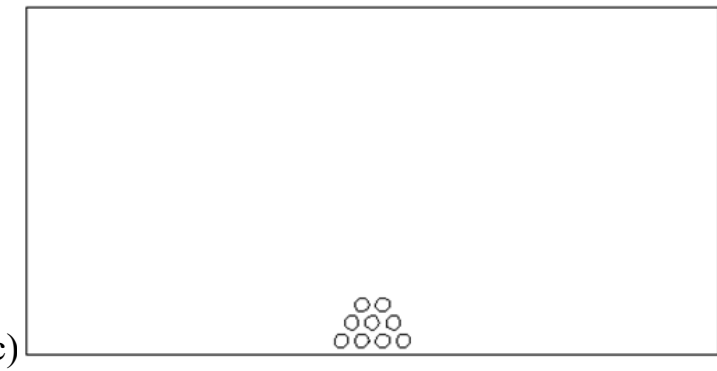

(d)

(e)

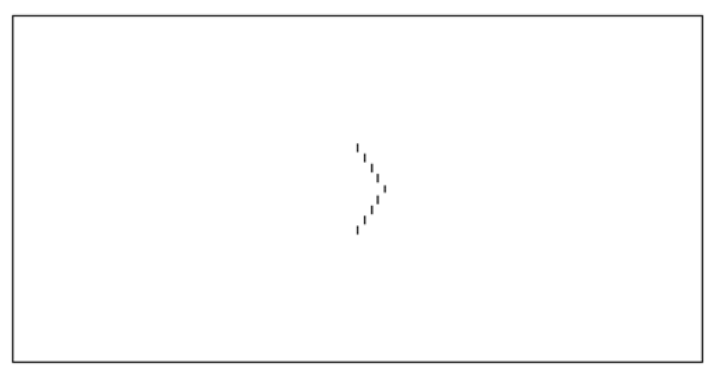

141 Fig. 1. Array configurations: (a) front view of Array-A; (b) front view of Array-B; (c) front view of Array-C; (d) top view of Array-A in 'zigzag'; (e) top view of Array-A in 'V-form'. 
For each array configuration, we first investigate the 'non-staggered' case with various values of $K$ (uniform across all discs) to find the optimal $K$ value to maximise the average power of all discs. In this study we do not consider a non-uniform distribution of $K$ across discs; however, Hunter et al. (2015) has shown that varying $K$ across a non-staggered array of four and eight discs does not increase the maximum average power. The maximum average power obtained here may therefore be taken as the upper limit of power extraction by the non-staggered array. Then we investigate the effect of staggering (or streamwise gap) with keeping this optimal $K$ value obtained for the non-staggered case. It should be noted that, strictly speaking, this $K$ value may not be optimal for the staggered cases. However, as will be shown later, the average power of the discs tends to change only slightly for a relatively wide range of $K$ around its optimal value. Therefore we can consider that the power of the staggered arrays calculated from the above procedure also corresponds approximately to the upper limit of power extraction.

In addition to these array configurations summarised in Table 1, we have also tested a large array consisting of 25 discs in order to demonstrate the effect of the number of discs; see Appendix for further details of this additional case.

\subsection{Flow parameters and boundary conditions}

In the earlier numerical study by Nishino and Draper (2015) three different inflow conditions have been tested, namely: (i) uniform inflow with a low freestream-turbulence (FST) level, (ii) vertically sheared inflow, and (iii) uniform inflow with a high FST level (as high as the sheared inflow case at the location of the discs). In the present study, we consider only the uniform inflow with a low FST level. This is mainly because the earlier study has shown that the power increase of a lateral array of actuator discs is very similar between the three different inflow cases, although another reason for employing a uniform inflow is to make a fair comparison between the Array-A, Array-B and Array-C cases. It should be noted that, in a more realistic case with vertically sheared inflow, the Array-B and 
to be insensitive to the shear of inflow if the power coefficient is defined using the average of the cube of the upstream velocity of the fluid passing through the disc (Draper et al. 2016).

Throughout the study, the inflow velocity is fixed at $U_{r e f}=10 \mathrm{~m} / \mathrm{s}$. In addition, the inlet values for $k$ and $\varepsilon$ are fixed at $k_{i n}=1.5 \times 10^{-4} \mathrm{~m}^{2} / \mathrm{s}^{2}$ and $\varepsilon_{i n}=3.02 \times 10^{-7} \mathrm{~m}^{2} / \mathrm{s}^{3}$, respectively, following the earlier study by Nishino and Draper (2015). Symmetry conditions are applied at the side boundaries of the domain, whereas slip-wall conditions are employed for the top and bottom boundaries; hence the uniform velocity profile given at the inlet is maintained throughout the domain (unless the discs perturb the flow). The values of $k$ and $\varepsilon$ given at the inlet gradually decrease towards downstream without mean shear to maintain the turbulence level, resulting in a relatively low turbulent viscosity ratio of $\mu_{T} / \mu=420$ at the centre of the domain (where the discs are placed). For the outlet boundary, zero streamwise-gradient conditions are prescribed for the velocities, $k$ and $\varepsilon$, with a constant gauge pressure of $0 \mathrm{~Pa}$. The Reynolds number based on the disc diameter is 67 million.

\subsection{Computational grids}

The computational grids have been created using the mesh extrusion function available in ANSYS ICEM. Specifically, for each array configuration a 2D multi-block structured grid was created first for a cross-section of the domain and then this $2 \mathrm{D}$ grid was extruded for the length of the domain in the streamwise direction, resulting in a 3D multi-block structured grid. A snapshot of a 2D cross-section grid for Array-B is shown in Fig. 2 as an example.

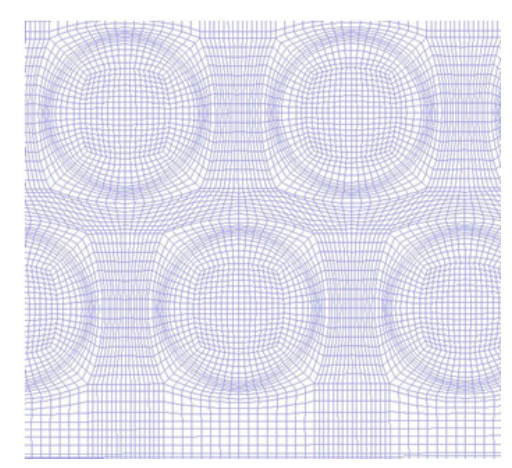

Fig. 2. Cross-sectional view of the computational grid for Array-B. 

an appropriate number of cells for the study. For the mesh used in the main part of the study, the minimum cell dimension is $0.0015 D$ near the disc edge (to resolve the steep shear flow around each disc sufficiently). The circumference of each disc is divided into 64 cells to represent with sufficient accuracy the round shape of the disc, whereas the spanwise length (z-direction) and height $(y$ direction) of the 2D cross-section are divided into 245 and 105 elements, respectively. For the extrusion of the 2D grid in the streamwise $(x)$ direction, grid points are allocated non-equidistantly; the minimum streamwise cell size is $0.002 D$ near the discs and the maximum size is about $1 D$ near the inlet and outlet boundaries. The total number of cells in the streamwise direction varies from 160 for the non-staggered cases to 175 for the staggered cases. The resultant total number of cells for the 3D grid varies between 5.3 and 6 million, depending on the array configuration.

205

\section{Results}

\subsection{Mesh sensitivity analysis}

We have performed a mesh sensitivity analysis for non-staggered Array-B (with $K=2.5$ for all 9 turbines). Four different grids were tested with an increasing number of mesh elements from 4 million to 7.5 million. Figure 3 shows the effect of the number of elements on the axial induction factor $a$, which is calculated for each turbine as

$$
a=1-\frac{\left\langle U_{d}\right\rangle}{U_{r e f}}
$$

where $\langle\phi\rangle$ denotes the average of a variable $\phi$ over the disc. In the figure, 'mid' shows the value of $a$ for the middle turbine (in the first row), whereas 'average' shows the average value of $a$ for all 9 turbines. As can be seen from the figure, the impact of the mesh resolution on the disc averaged axial induction factor (and also on the disc thrust and power, which are not presented here for brevity) is negligibly small for the grids with more than 5 million elements. This number of elements compares well with the earlier study by Nishino and Draper (2015), who also conducted a mesh sensitivity study and eventually employed a grid with about 5.3 million mesh elements for a non-staggered single row of 9 discs. 


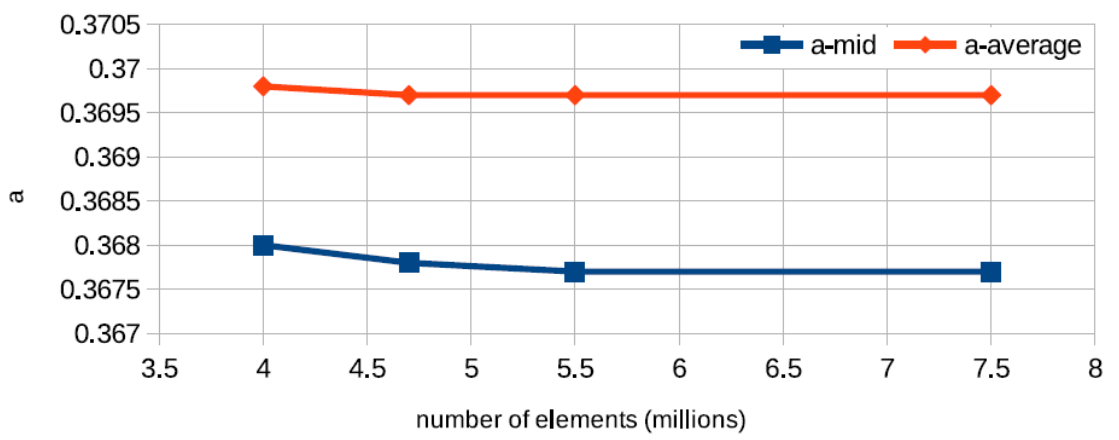

222

223

Fig. 3. Effect of the number of mesh elements on the axial induction factors for non-staggered Array-B ('mid': middle turbine in the first row; 'average': average of all 9 turbines).

\subsection{Non-staggered single row of up to 9 discs}

In this section we investigate the influence of the number of turbines on the limit of power extraction by a non-staggered single row (Array-A), although this configuration has already been studied earlier by Nishino and Draper (2015). We have performed simulations for 1, 3, 5, 7 and 9 turbines and, for each case, we have tested several different $K$ values to maximise the power coefficient $C_{p}$, which is calculated as

$$
C_{p}=\frac{\int M_{x} U_{d} \mathrm{~d} A}{\frac{1}{2} \rho U_{r e f}^{3} A}=K \frac{\left\langle U_{d}^{3}\right\rangle}{U_{r e f}^{3}}
$$

where $A$ is the area of each disc. For the single disc case $K=2$ was found to maximise $C_{p}$ (as can be predicted from the Betz theory), whereas for all other cases $K=2.5$ was found to give a higher $C_{p}$ value. Figure 4 shows the effect of the number of turbines on the maximum value of $C_{p}$ (again for the middle turbine and for the average of all turbines). Note that, for the single disc case, the $C_{p}$ value obtained is slightly higher than the Betz limit (0.593); this slight difference is due to the effect of the ground (which provides a weak partial blockage effect) as well as to the effects of the viscosity and three-dimensionality of the flow. The maximum $C_{p}$ value increases with the number of turbines in the array due to the local blockage effect. These results agree very well with the results reported earlier by Nishino and Draper (2015). 


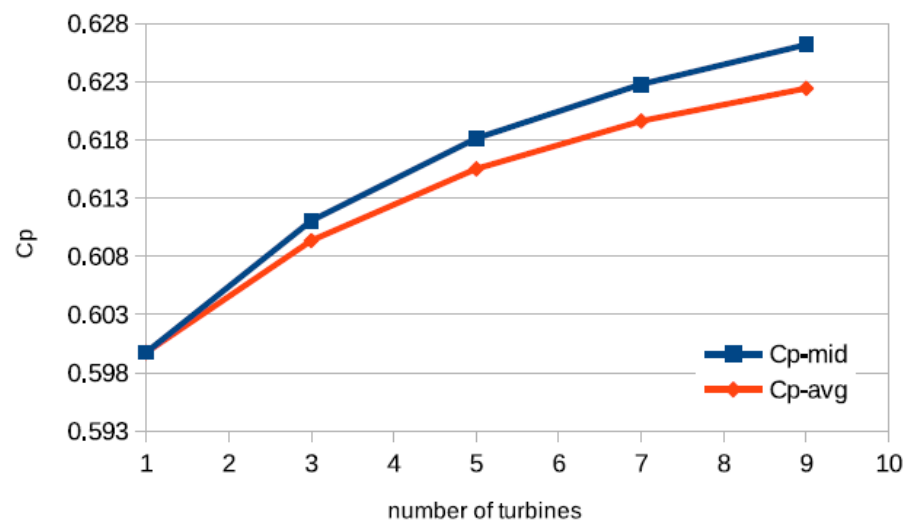

Fig. 4. Effect of the number of turbines on the maximum power coefficient for non-staggered

Array-A ("mid": middle turbine; "avg": average of all turbines).

Figure 5 shows contours of streamwise velocity normalised by the undisturbed flow speed $\left(U_{\text {ref }}=10 \mathrm{~m} / \mathrm{s}\right)$ for the non-staggered single row of $1,3,5,7$ and 9 discs, plotted at the height of the centre of the discs. It can be seen how the flow around the discs changes with the number of discs in the array. In particular, we can observe two main features of the flow affected by the local blockage: flow deceleration in front of the entire array increases with the number of discs.

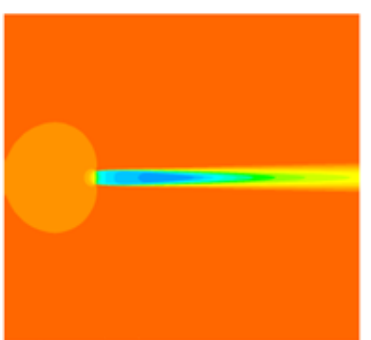

(a) Isolated turbine

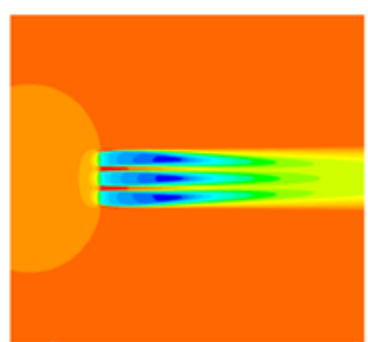

(b) Array of 3 turbines

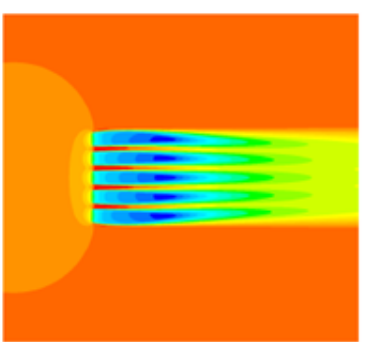

(c) Array of 5 turbines

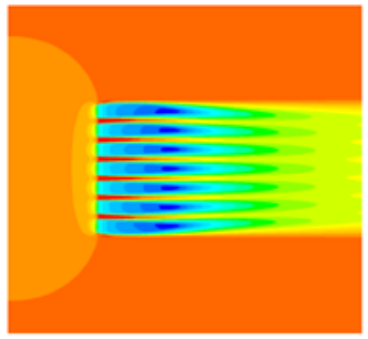

(d) Array of 7 turbines

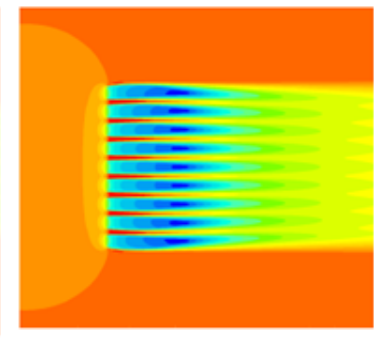

(e) Array of 9 turbines

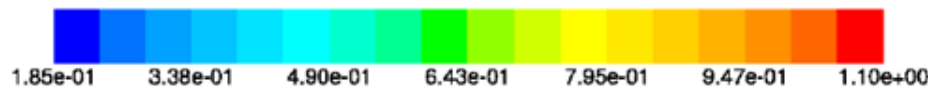

Fig. 5. Contours of streamwise velocity (normalised by the inlet velocity $U_{r e f}=10 \mathrm{~m} / \mathrm{s}$ ). 


\subsection{Non-staggered double and triple rows}

258 Next, we investigate potential benefits of arranging turbines not only in the spanwise direction but also in the vertical direction (Array-B and Array-C). As noted earlier, these configurations are not conventional but follow the concept of 'multi-rotors', studied recently by Jamieson and Branney (2012) and Manwell et al. (2014). Note that here we again consider 9 turbines with the same intraturbine spacing of $0.5 D$, but these 9 turbines are divided into two rows (for Array-B) or three rows (for Array-C) as depicted in Fig. 1.

Figure 6 shows the power coefficient values obtained for non-staggered Array-B and Array-C with different $K$ values (note that all 9 discs have the same $K$ value in each simulation). As can be seen from the figure, for both Array-B and Array-C, the power is maximised around $K=2.5$, similarly to the single row case (Array-A) discussed in the previous section. Moreover, the maximum power coefficient values for these two array configurations are only slightly higher than that for Array-A, indicating that the benefit of local blockage can be enhanced only slightly by dividing the 9 turbines into two or three rows. Interestingly, the amounts of power that can be extracted by Array-B and Array-C are almost identical; the reason for this will be discussed later in Section 4.

(a)

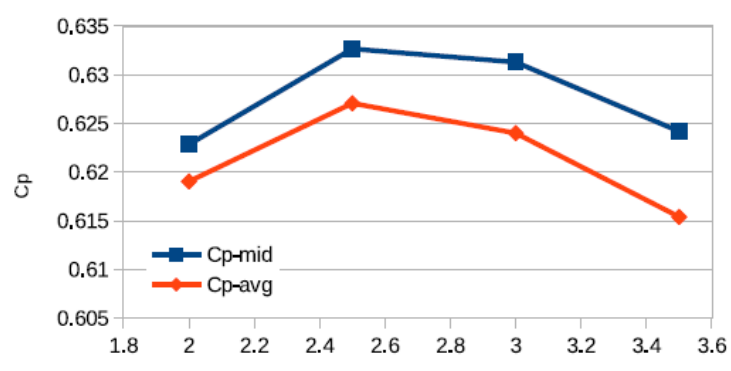

\subsection{Effect of staggering}

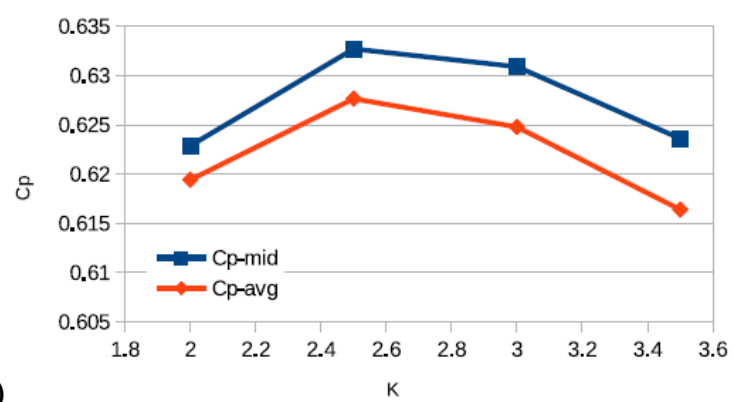

(b)

Fig. 6. Power coefficient for non-staggered multi-row arrays: (a) Array-B; (b) Array-C.

Now we investigate the effect of staggering the array in the streamwise direction. We consider three different array configurations, namely 'zigzag' Array-A, 'V-form' Array-A and 'step' Array-B, as 
summarised earlier in Table 1. The main interest here is the effect of the streamwise gap between two neighbouring turbines on the maximum power extracted by the array. All results presented below are for $K=2.5$ for all turbines. This $K$ value is nearly optimal and gives a power coefficient value very close to its maximum for each array configuration investigated here, although the exact optimum $K$ value depends on the array configuration and tends to gradually increase with the maximum power extracted by the array.

Figure 7 shows the effect of the streamwise gap on the maximum power coefficients for the 'zigzag' Array-A. Note that three different power coefficient values are plotted for each case: 'first row' (average of 5 discs in the upstream row), 'second row' (average of 4 discs in the downstream row) and 'average' (average of all 9 discs). It can be seen that the power extracted by the second row is maximised when the streamwise gap between the two rows is $1 D$ to $1.5 D$. This is because turbines in the second row are located in the flow accelerated locally due to the turbines in the first row, as shown in Fig. 8. However, this power increase in the second row is accompanied by a power decrease in the first row, resulting in a slight decrease in the total power. Importantly, the results show that the total power extracted by the staggered Array-A is always lower than that extracted by the nonstaggered Array-A regardless of the streamwise gap. This agrees with the recent numerical study by Hunter et al. (2015) for a similar cross-stream array of tidal turbines.

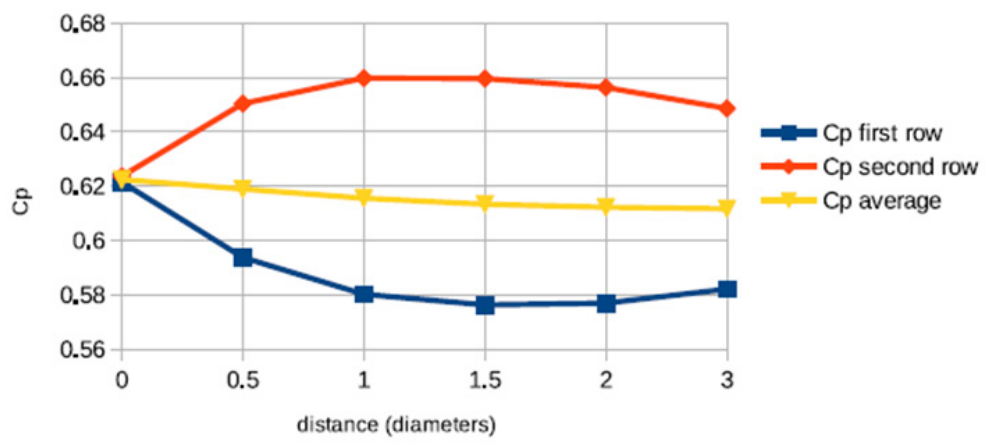

Fig. 7. Effect of the streamwise gap on the maximum power coefficient (zigzag Array-A). 


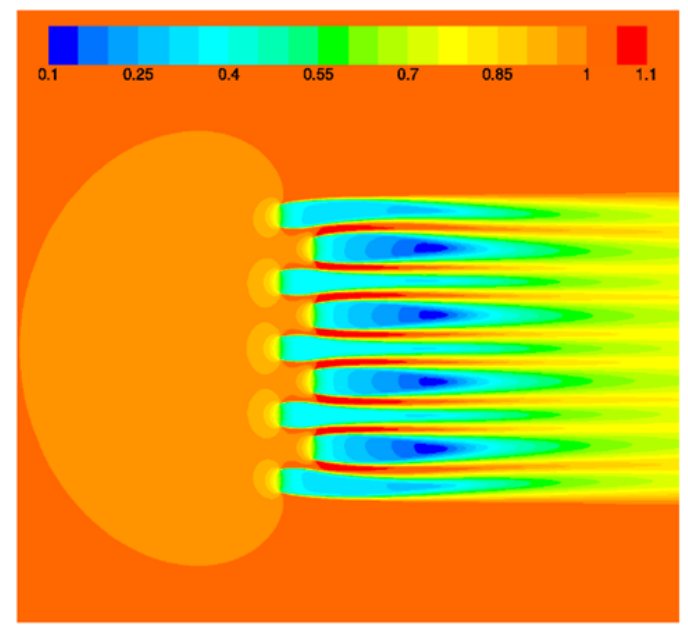

Fig. 8. Contours of normalised streamwise velocity (Array-A with $1.5 D$ streamwise gap).

302

Figure 9 presents results obtained for the staggered (or 'step') Array-B, again for the 'first row' (average of 5 discs in the upstream row), 'second row' (average of 4 discs in the downstream row) and 'average' (average of all 9 discs). The results are very similar to the 'zigzag' Array-A case described above; staggering the array increases the power extracted by the second row but decreases the power extracted by the first row, resulting in a slight decrease in the total power. Figure 10 shows contours of normalised streamwise velocity for the staggered Array-B with $1.5 \mathrm{D}$ gap, plotted at two different vertical positions corresponding to the disc centre for the first and second rows, respectively. located in the flow accelerated locally due to the turbines in the first row.

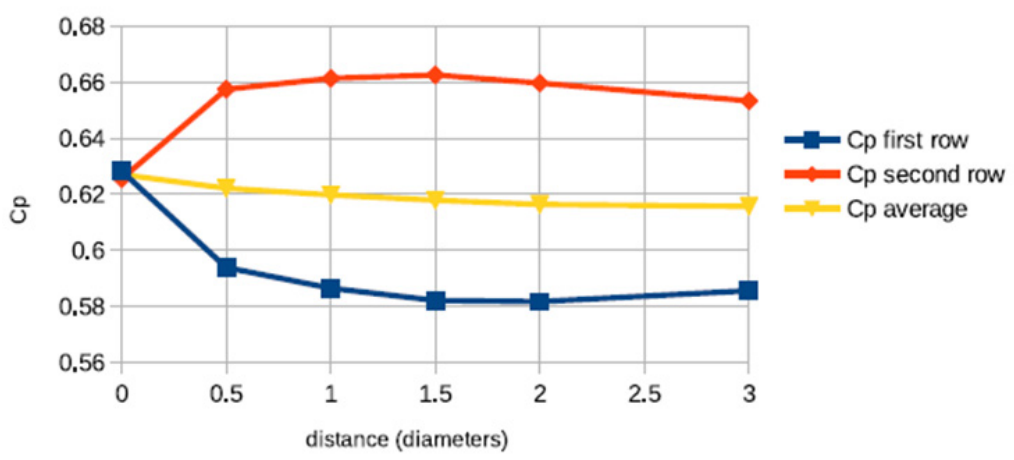

Fig. 9. Effect of the streamwise gap on the maximum power coefficient (staggered Array-B). 


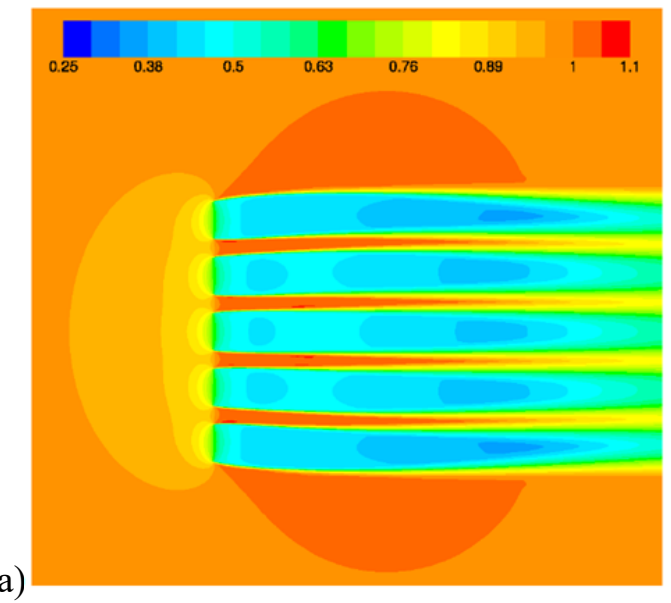

(b)

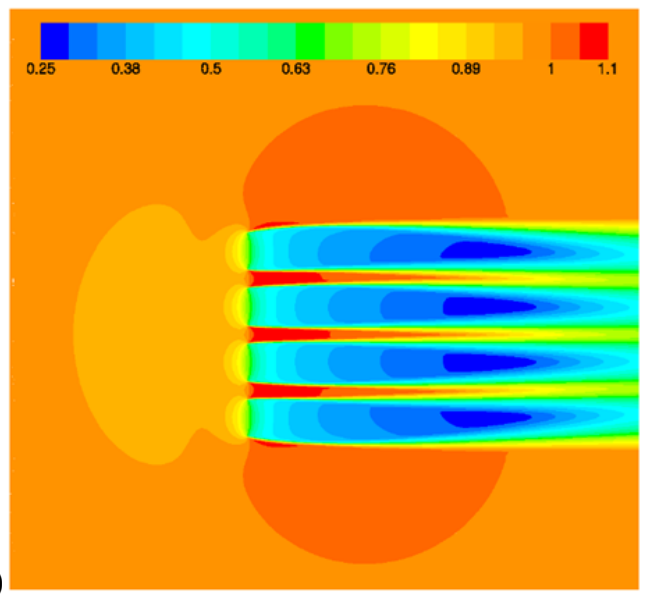

Fig. 10. Contours of normalised streamwise velocity (Array-B with $1.5 D$ streamwise gap): (a) at the 'hub-height' for the first row; (b) at the 'hub-height' for the second row.

Finally, Fig. 11 shows results for the V-form Array-A. Here the maximum power coefficient values are plotted for the 'most upstream' turbines (located at the spanwise ends of the array), 'most downstream' turbine (located at the middle of the array) and the average of all 9 turbines. Again the trend is very similar to the 'zigzag' Array-A and 'step' Array-B; the total power extracted by the array decreases as we increase the streamwise gap between neighbouring turbines, although the power extracted by the most downstream turbine can be substantially higher. The variations of the power coefficient across a half of the array (due to symmetry) are plotted in Fig. 12 for the six different streamwise gap cases tested. It can be seen that the power increases substantially only for the middle turbine and decreases for the majority of other turbines in the array.

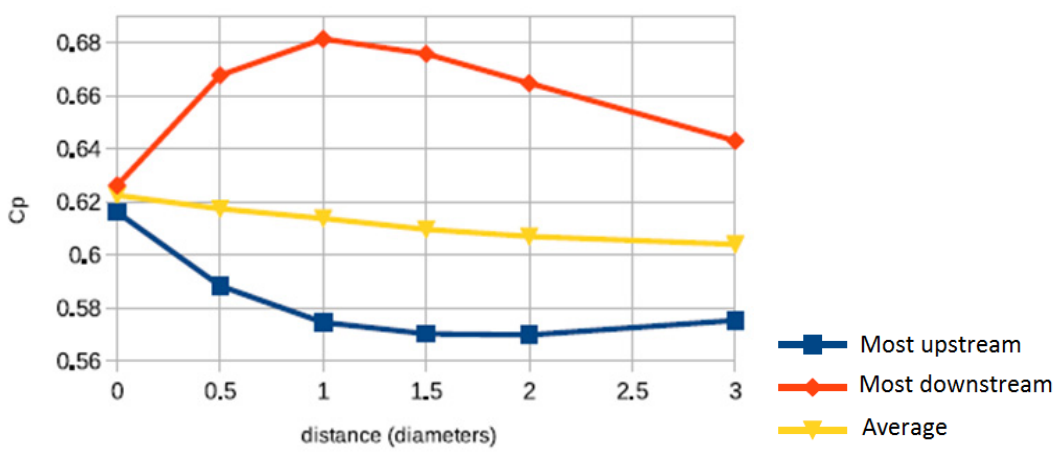

Fig. 11. Effect of the streamwise gap on the maximum power coefficient (V-form Array-A). 


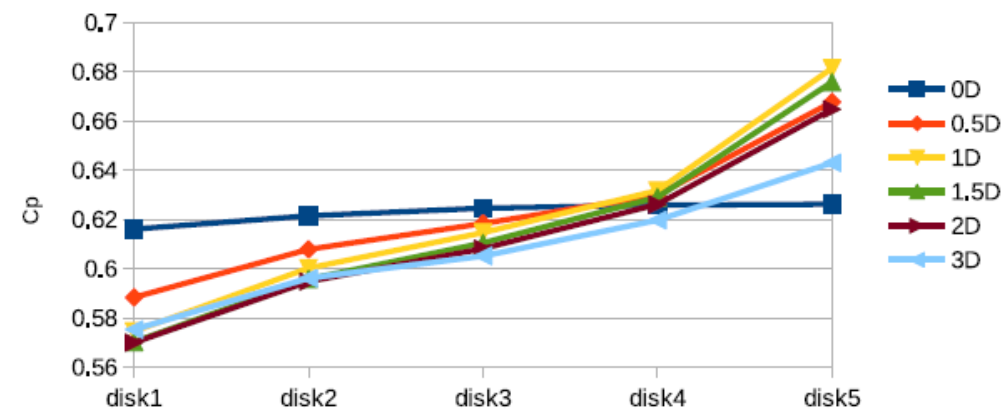

Fig. 12. Effect of the streamwise gap on the variation of power coefficient across V-form Array-A ('disk 5' is the middle turbine, which is located most downstream).

\section{Discussion and conclusions}

In this study we have considered three different types of dense wind turbine array configurations, namely Array-A, Array-B and Array-C. For Array-A and Array-B, we also considered staggering the array in the streamwise direction. In this section we discuss the performance of non-staggered arrays first and then the effect of staggering the array, followed by some conclusions.

\subsection{Non-staggered array performance}

For the non-staggered cases, our numerical results have shown that the limit of power extraction by a dense spanwise array of 9 turbines can be about $5 \%$ higher than that by isolated turbines, confirming the results reported earlier by Nishino and Draper (2015). We have also tested a new idea of arranging turbines not only horizontally but also vertically (following the 'multi-rotor' concept) by dividing the 9 turbines into 2 rows (Array-B) or 3 rows (Array-C); however, the limit of power extraction by these unconventional arrays was only slightly (less than $1 \%$ ) higher than that by the single spanwise array (Array-A). Moreover, the performance of Array-B and Array-C was found to be almost identical. A possible explanation for the above results can be made by considering the level of local blockage experienced by each turbine in these three arrays. As depicted in Fig. 13, we can classify the turbines in these arrays into the following three types:

(i) Turbines with a 'high blockage' level (red): these turbines are completely $\left(360^{\circ}\right)$ surrounded by other turbines or the ground; 
(ii) Turbines with a 'medium blockage' level (green): these turbines are mostly $\left(\sim 240^{\circ}\right)$ but not completely surrounded by other turbines or the ground; and

(iii) Turbines with a 'low blockage' level (blue): these turbines are only partially $\left(\sim 180^{\circ}\right)$ surrounded by other turbines or the ground.

It can be seen that the types of turbines in Array-B and Array-C are exactly the same (3 'high', 4 'medium' and 2 'low' blockage levels), which may explain the almost identical limit of power extraction by these two arrays. This explanation also suggests that a possible way to further increase the limit of power extraction (per turbine) is to arrange more turbines and thereby increase the proportion of 'high blockage' turbines in the array. An example of this will be presented in the Appendix.

It is worth noting that the above classification of the level of blockage (high, medium and low) considers only the range of directions surrounded by other turbines or the ground and does not consider the type of the surrounding turbines. Theoretically, a 'high blockage' turbine surrounded by other 'high blockage' turbines may experience a more significant local blockage effect than a 'high blockage' turbine surrounded by 'low blockage' turbines, due to the array-size effect (Nishino and Willden, 2013). It should also be noted that, in general, the local blockage effect depends significantly on the intra-turbine spacing, which has been fixed at $0.5 \mathrm{D}$ in this study.

(a)

\section{$\bullet$}

(c)

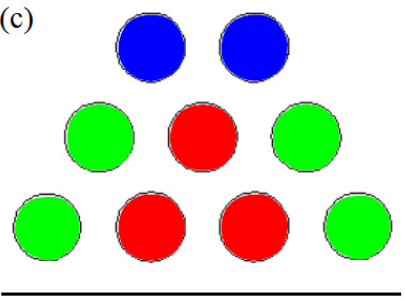

(b)
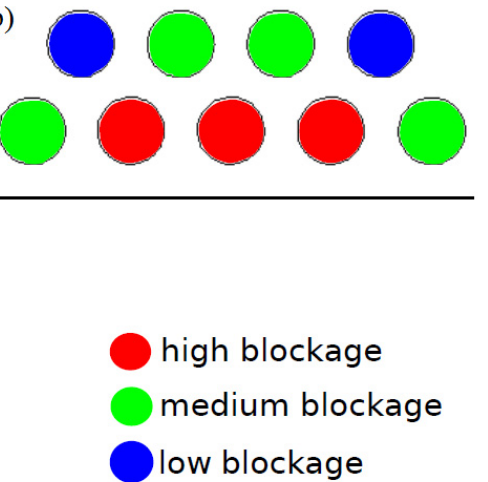

Fig. 13. Local blockage level for each turbine: (a) Array-A; (b) Array-B; (c) Array-C. 


\subsection{Staggered array performance}

378 As for the effect of staggering the array, our results have shown that the limit of total power extraction by a dense cross-stream array cannot be increased further by staggering the array in the streamwise direction. This agrees with the recent finding by Hunter et al. (2015), who performed similar RANS simulations of seven porous discs placed in a shallow water channel (to represent tidal turbines). This agreement suggests that, although in general the effect of local blockage tends to be less significant for wind turbines than for tidal turbines due to the lack of flow confinement above the turbines, the relationship between 'local blockage' and 'local flow acceleration' is still the same, i.e. staggering the array in the streamwise direction will enhance the power of downstream turbines due to the effect of local flow acceleration but reduce the power of upstream turbines as the effect of local blockage diminishes, resulting in a reduction of total power. This also agrees with more recent results reported by Zanforlin and Nishino (2016), who performed 2D unsteady RANS simulations of two counterrotating vertical-axis turbines with various intra-turbine spacing and wind directions, showing that the total power of two vertical-axis turbines is maximised when the turbines are placed side-by-side with respect to the wind direction.

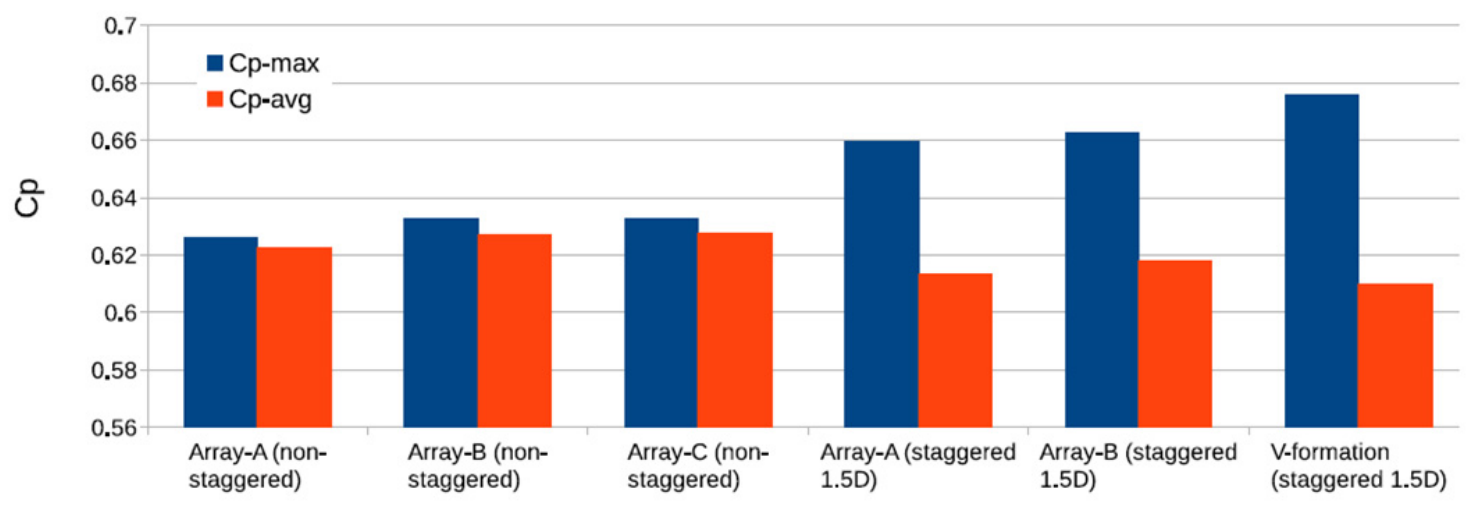

Fig. 14. Comparison of power coefficients for different array configurations.

Figure 14 summarises the limit of power extraction predicted for six representative array configurations tested in this study (with $K=2.5$ ). Note that 'Cp-max' shows the power coefficient of the turbine extracting the highest power compared to other turbines in the array, whereas ' $\mathrm{Cp}$-avg' 
shows the average power coefficient for all 9 turbines in the array (and therefore indicates the total power). It can be seen that the power of a particular turbine can be enhanced but the total power of the array is reduced by staggering the array in the streamwise direction.

\subsection{Conclusions}

In conclusion, we have investigated numerically, using 3D RANS simulations of up to 9 porous discs, how the limit of power extraction by a given number of wind turbines could be increased by arranging them densely in the cross-stream direction and thereby utilising the so-called 'local blockage effect'. The focus of the present numerical study, using a simple porous disc model instead of a more realistic rotor model, is limited to the 'limit' of power extraction (corresponding to the Betz limit for the case of isolated turbines); however, similar effects of blockage due to neighbouring turbines have also been observed experimentally by McTavish et al. (2015) using more realistic (but a smaller number of) wind turbine models. Our numerical results have demonstrated that:

(i) The increase in the limit of power extraction due to the local blockage effect tends to be enhanced by increasing the number of turbines in the array;

(ii) When the number of turbines in the array is relatively small (e.g. 9 turbines), the limit of power extraction can be only slightly enhanced further by arranging the turbines vertically as well as horizontally (so-called 'multi-rotor' configuration), since only a small number of turbines in the array can experience a higher level of blockage by doing so; and

(iii) The limit of total power extraction by a dense cross-stream array tends to decrease by staggering the array in the streamwise direction, although some of the turbines in the array may extract more power due to the effect of local flow acceleration.

Further investigations with a larger number of turbines, and a more realistic turbine model, would be required in future studies in order to fully understand the effect of local blockage for wind turbines; however, such investigations would require huge computational resources and/or a large experimental facility. 


\section{$427 \quad$ Appendix}

428 To further demonstrate the influence of the number of turbines in the array, we have performed some additional simulations with 25 discs arranged in 3 rows $(8,9$ and 8 discs in the first, second and third rows, respectively; see Fig. A.1(a)). All computational conditions are the same as those employed in the main body of the paper, except that the diameter of each disc $(D)$ is $60 \mathrm{~m}$ (instead of $100 \mathrm{~m})$ in these additional simulations to maintain the same global blockage ratio $\left(B_{G} \approx 0.006\right)$ as the 9 -disc cases investigated earlier (to make a fair comparison). The results are shown in Fig. A.1(b) in terms of the maximum and average power coefficient values. Compared to the results for the 9-disc Array-B and Array-C cases presented earlier in Fig. 6, it is clear that the power coefficient for this 25-disc case is substantially higher, demonstrating the impact of the number of turbines in the array on the limit of power extraction by the array. As discussed by Nishino and Draper (2015), the upper limit of the power coefficient of each turbine in this type of dense cross-stream array is expected to increase up to about 0.8 as we further increase the number of turbines in the array. It should be remembered, however, that the power coefficient discussed here has been defined for each turbine, using the rotor swept area as the reference area. If the power coefficient of an entire array is considered and defined using the entire array area (i.e. including the gap area between rotors as well as the rotor swept area), it is unlikely that this power coefficient will exceed the Betz limit (0.593) regardless of the number of turbines in the array or the intra-turbine spacing.

(a)

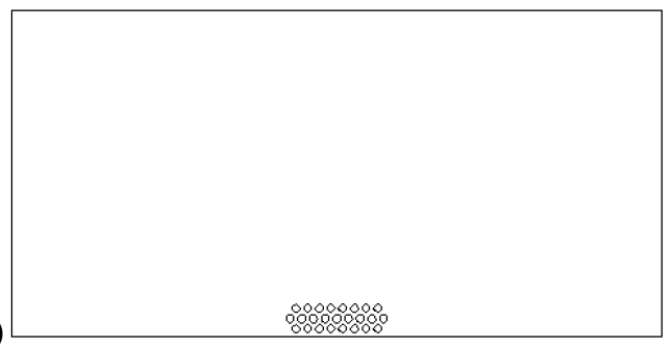

(b)

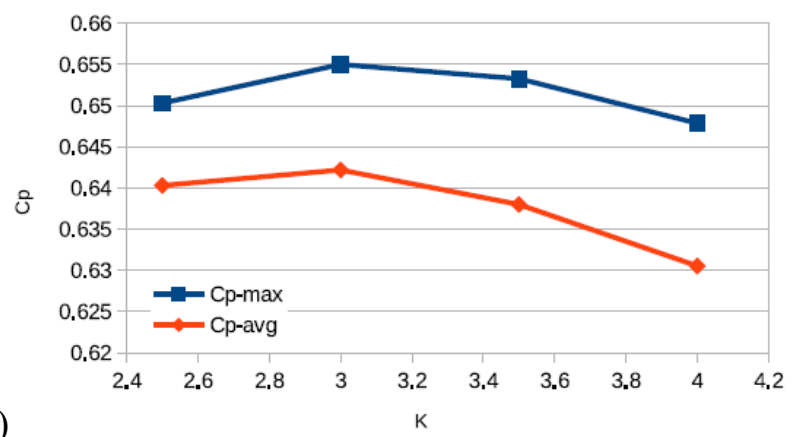

447 Fig. A.1. Additional simulations with 25 discs: (a) front view of the array; (b) power coefficient. 


\section{References}

450 Chasapogiannis, P., Prospathopoulos, J.M., Voutsinas, S.G., Chaviaropoulos, T.K., 2014. Analysis of

451

452

453

454 the aerodynamic performance of the multi-rotor concept. Journal of Physics: Conference Series 524, 012084.

Draper, S., Nishino, T., 2014. Centred and staggered arrangements of tidal turbines. Journal of Fluid Mechanics 739, 72-93.

Draper, S., Nishino, T., Adcock, T.A.A., Taylor, P.H., 2016. Performance of an ideal turbine in an inviscid shear flow. Journal of Fluid Mechanics 796, 86-112.

Garrett, C., Cummins, P., 2007. The efficiency of a turbine in a tidal channel. Journal of Fluid Mechanics 588, 243-251.

Hunter, W., Nishino, T., Willden, R.H.J., 2015. Investigation of tidal turbine array tuning using 3D Reynolds-averaged Navier-Stokes simulations. International Journal of Marine Energy 10, $39-51$.

Jamieson, P., Branney, M., 2012. Multi-rotors; a solution to 20MW and beyond? Energy Procedia 24, $52-59$.

Launder, B.E., Spalding, D.B., 1974. The numerical computation of turbulent flows. Computer Methods in Applied Mechanics and Engineering 3(2), 269-289.

Manwell, J.F., McGowan, J.G., Brena, S., Verma, P., 2014. A comparative study of a three rotor and a single rotor $5 \mathrm{MW}$ wind turbine based on economic and structural considerations. Wind Engineering 38(6), 643-657.

McTavish, S., Rodrigue, S., Feszty, D., Nitzsche, F., 2015. An investigation of in-field blockage effects in closely spaced lateral wind farm configurations. Wind Energy 18(11), 1989-2011.

Nishino, T., 2016. Two-scale momentum theory for very large wind farms. Journal of Physics: Conference Series 753, 032054.

Nishino, T., Draper, S., 2015. Local blockage effect for wind turbines. Journal of Physics: Conference Series 625, 012010. 
475 Nishino, T., Willden, R.H.J., 2012a. Effects of 3-D channel blockage and turbulent wake mixing on the limit of power extraction by tidal turbines. International Journal of Heat and Fluid Flow $37,123-135$.

478

Nishino, T., Willden, R.H.J., 2012b. The efficiency of an array of tidal turbines partially blocking a 479 wide channel. Journal of Fluid Mechanics 708, 596-606.

480

Stevens, R.J.A.M., 2016. Dependence of optimal wind turbine spacing on wind farm length. Wind

Zanforlin, S., Nishino, T., 2016. Fluid dynamic mechanisms of enhanced power generation by closely spaced vertical axis wind turbines. Renewable Energy 99, 1213-1226. 